\title{
Metabolic surgery pave the way towards curing diabetes 2 in the globe
}

\section{Editorial}

It has been a stimulating trigger for all those involved in the management of Diabetes two to find an exit from this insulting disease as it is called with its harming effect on all body systems. For many decades medical treatment of Diabetes 2 (Insulin Resistant Diabetes) consisted support, advices, dieting, followed by oral hypoglycemic medication to end eventually in administrating Insulin, initially the use of insulin was reserved to acute cases and when blood sugar goes out of control, in the form of Insulin Act rapid, then there was various modifications ended in long acting Insulin and Human Insulin etc. However we were as physicians and surgeons not impressed and frankly we were not convinced with the outcome of various conservative methods as could not achieve more than maintaining blood sugar level within acceptable range, despite the variety of advices and treatment we were far from getting any real cure for this disease and with all sorrow we probably only been able to delay those systemic complication but not cure it, and all our attempts were classified as palliative no more. In the not too distant past, the role of the surgeons of various specialties was limited to local debridement of devitalized gangrenous tissue and amputation, despite all the enthusiasm by vascular surgeons, the results of reconstructive and interventional procedures proved not encouraging as most of the vascular trees are diseased or even calcified. The other systems also were less luckier to achieve any cure. We just accepted to be less effective in this field, over all we as physicians with human ambition been waiting for a star to glow our road to better action towards our failure. The sunray eventually shine on the globe with the encouraging results achieved from Bariatric Surgery in combating obesity , $80 \%$ of those obese patients will acquire Diabetes in the process of this disease .

With the era of Bariatric Surgery, it became obvious that those with diabetes will benefit from this type of surgery in $80 \%$ of treated cases R\&Y Gastrectomy. This achievements stimulated investigators to go in analyzing this results of Bariatric Surgery and its impact on resolving diabetes. Furthermore a question was raised whether this procedure are applicable to non obese diabetic patients whom their $\mathrm{BMI}<40$, all of us were encouraged to take further step towards applying the same surgical procedures to those diabetics with BMI around 35 and even with those of $\mathrm{BMI}<30$, the results was excellent and diabetes faded. From now on those surgeons involved got in detailed research to develop a modified approach called Metabolic

\author{
Volume 4 Issue 3 - 2017
}

\author{
Kubtan MA \\ Head of Dept of Surgery, Syrian Private University, Syria \\ Correspondence: Kubtan MA, MD-FRCS, Head of \\ Department of Surgery, Medical Faculty, Syrian Private \\ University, Damascus, Syria, Email makubtan@gmail.com
}

Received: April 27, 2017| Published: May 01, 2017

Surgery designed specifically for Diabetes 2 in the form of Pancreatic Duodenal Diversion an Duodenal switch. To get in details it was proved that preventing the consumed fatty food from meeting with alkaline Bile Salts in second part of Duodenum will keep it hydrophobic and unobservable in the gut, this will have its impact on the entero-hepatic circulation in reducing fat absorption and blocking function upon Insulin metabolism in other word freeing Insulin to restore its physiological function and transferring Glucose molecule inside hepatic cells to be metabolized.

Now we can say that we are faced with revolutionary prospect in treating diabetes 2.DSS-II, Diabetic Surgery Summit-II and $3^{\text {rd }}$ World Congress on Interventional Therapies for Type 2 Diabetes Convened in London, U.K., 28-30 September 2015, were numerous randomized clinical trials reviewed from all over international centers and societies, all came to the conclusion that time now for Metabolic Surgery to drive the way against diabetes 2 with confidence. In this summit endorsed the DSS-II consensus statements and guidelines were approved from over 48 international authorities and societies involved with management of Diabetes. Now we can advice our colleagues to rest assured that their patients will benefit from Metabolic Surgery, and we can recommend to our Diabetic patients that there is a glowing light at the end of the tunnel.

\section{Acknowledgements}

None.

\section{Conflict of interest}

The author declares no conflict of interest. 